\title{
REVIEW
}

\section{The Technical and Clinical Features of 3D-FLAIR in Neuroimaging}

\author{
Shinji NAGANAWA* \\ Department of Radiology, Nagoya University Graduate School of Medicine \\ 65 Tsurumai-cho, Shouwa-ku, Nagoya 466-8550, Japan
}

(Received November 25, 2014; Accepted January 28, 2015; published online March 31, 2015)

In clinical MR neuroimaging, 3D fluid-attenuated inversion recovery (3D-FLAIR) with a variable-flip-angle turbo spin echo sequence is becoming popular. There are more than 100 reports regarding 3D-FLAIR in the PubMed database. In this article, the technical and clinical features of 3D-FLAIR for neuroimaging are reviewed and summarized. 3D-FLAIR allows thinner slices with multi-planar reformation capability, a higher flow sensitivity, high sensitivity to subtle $T_{1}$ changes in fluid, images without cerebrospinal fluid (CSF) inflow artifacts, and a 3D dataset compatible with computer-aided analysis. In addition, 3D-FLAIR can be obtained within a clinically reasonable scan time. It is important for radiologists to be familiar with the features of 3D-FLAIR and to provide useful information for patients.

Keywords: magnetic resonance imaging, endolymphatic hydrops, 3D-FLAIR

\section{Introduction}

Fluid-attenuated inversion recovery (FLAIR) images are widely utilized in neuroimaging. Suppression of the cerebrospinal fluid (CSF) signal makes the recognition of various pathologies near the CSF space easier. ${ }^{1,2}$ 2D-FLAIR is used most often as part of routine clinical studies. 3D-FLAIR enables the acquisition of thinner slices without a significant CSF ghost artifact. ${ }^{3,4}$ The three-dimensional dataset obtained by 3D-FLAIR has the potential to be evaluated by computer-assisted quantitative post-processing analyses. ${ }^{5,6}$ Recent technical developments have shortened the acquisition time for 3D-FLAIR, facilitating use in a routine clinical setting as well as in specific clinical applications. Now, more than 100 papers can be found by a PubMed search for "3D-FLAIR". In this review, the technical features and clinical applications of 3D-FLAIR are reviewed and summarized for those who employ 3D-FLAIR imaging in clinical practice.

\footnotetext{
*Corresponding author, Phone: +81-52-744-2327, Fax: +8152-744-2335, E-mail: naganawa@med.nagoya-u.ac.jp
}

\section{Technical Features}

2D-FLAIR

2D-FLAIR was initially obtained using a conventional spin echo sequence. Despite a longer scan time, better lesion conspicuity by 2 D-FLAIR was expected, promising application to a wide range of diseases. ${ }^{7}$ Single-shot echo planar imaging (ss-EPI)-based 2D-FLAIR was reported to shorten the scan time dramatically; however, image distortion and low spatial resolution limited its utility. ${ }^{8}$ By utilization of a fast spin echo sequence, 2DFLAIR came into routine clinical practice. ${ }^{2}$

\section{$2 D$ vs $3 D$}

To acquire thinner slices, $3 \mathrm{D}$ acquisition is preferable, although $3 \mathrm{D}$ acquisition increases the scan time compared to $2 \mathrm{D}$ acquisition. In 2D-FLAIR, especially with thinner slices, CSF inflow artifacts can be a problem. ${ }^{3,4}$ FLAIR can sensitively detect subarachnoid hemorrhage (SAH); however, CSF artifacts can cause false-positive findings. ${ }^{9}$ 3DFLAIR allows the suppression of CSF-related artifacts as well as retrospective multi-planar slice reformatting (Fig. 1). ${ }^{3,10,11}$ One pitfall of 3D-FLAIR is the frequently obscured ivy sign in moyamoya disease. ${ }^{12}$ Another pitfall of 3D-FLAIR is less visibility for intra-arterial hyperintensity in patients 
$\mathbf{a}$

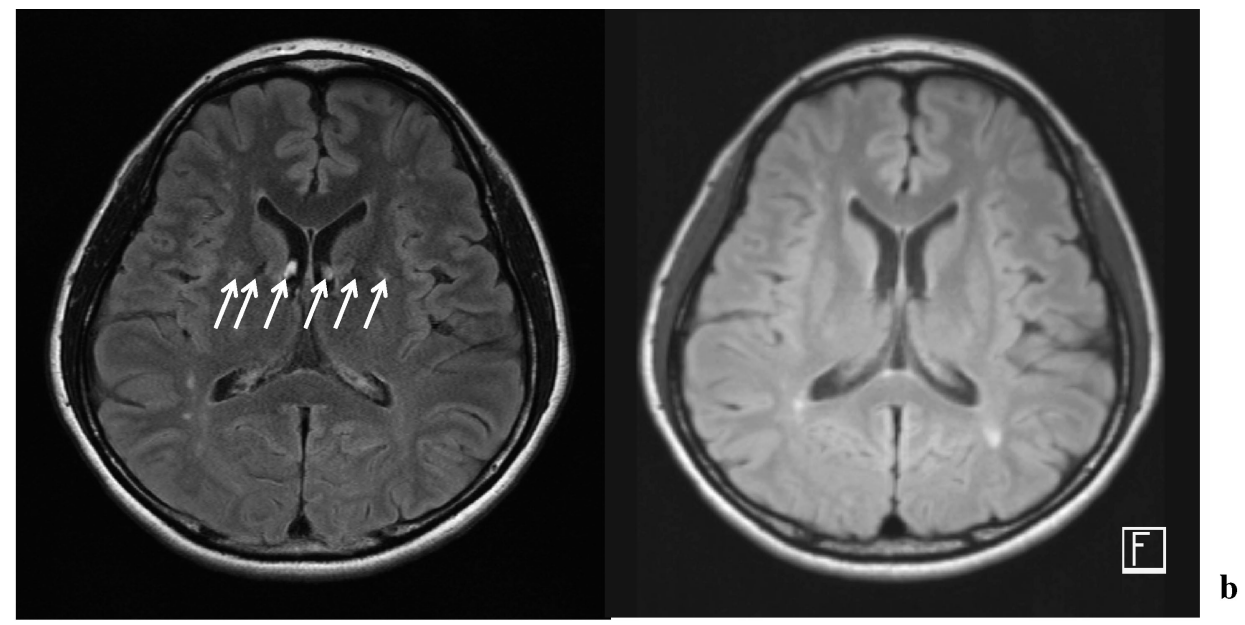

c

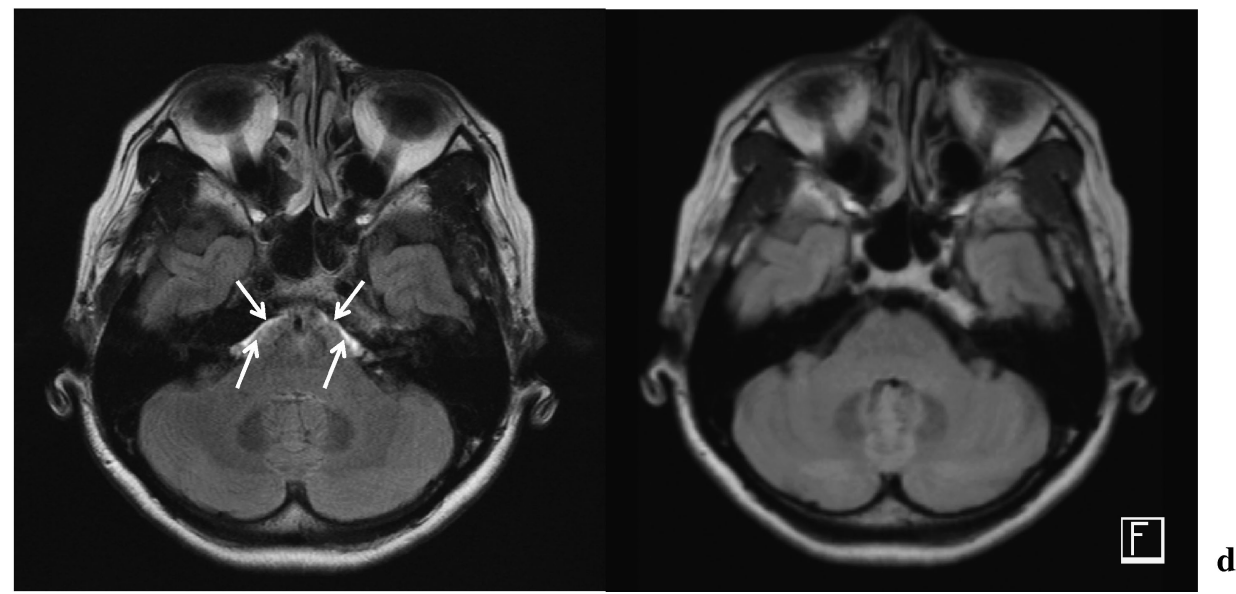

Fig. 1. Comparison of CSF-related artifacts on 2D- and 3D-FLAIR

3D-FLAIR images are reformatted to $4.5 \mathrm{~mm}$ thick, which are identical to the 2DFLAIR images. At the lateral-ventricle-level slice, CSF pulsation artifacts aligned in the phase encoding direction are seen on 2D-FLAIR (a) (arrows), but not on 3D-FLAIR (b). At the pons level, a CSF inflow artifact is seen on 2D-FLAIR (c) (arrows), but not on 3D-FLAIR (d).

with acute ischemic stroke (Fig. 2). ${ }^{13}$ In contrast, 2D-FLAIR sometimes suffers from an incomplete inversion pulse effect in areas disturbed by metallic substances. ${ }^{14}$ In a single-slab 3D-FLAIR covering the whole brain, a thicker inversion pulse or a nonspatially selective inversion pulse is utilized. Artifacts caused by metallic substances are usually weaker with 3D-FLAIR than with 2D-FLAIR (Fig. 3). ${ }^{14}$

\section{Single slab or multi-slab}

To cover the whole brain with a single slab, more than 160 slices are usually required. Using a conventional turbo spin echo (TSE) sequence with an echo train length (ETL) of around 30, scan time can exceed $1 \mathrm{~h}$. A multi-slab approach using a conventional TSE sequence improves the time efficiency. During the repetition time period, multiple slabs are excited sequentially. To acquire gapless slices, it is necessary to obtain more than two separate sets of interleaved multi-slabs. This requirement reduces the time efficiency. The multi-slab approach is a hybrid of $2 \mathrm{D}$ and $3 \mathrm{D}$ acquisitions, but multislab 3D-FLAIR is not currently used widely in clinical applications due to the slab boundary artifact in reformatted images. ${ }^{15}$

To shorten the scan time of single-slab 3DFLAIR, a variable-flip-angle turbo spin echo (VFATSE) sequence is usually employed. ${ }^{14-16}$ In this review, 3D-FLAIR by VFA-TSE is mostly discussed.

\section{Variable-flip-angle turbo spin echo}

To shorten the scan time in TSE imaging, increasing the echo train length is one solution. To suppress the blurring of anatomical details, the echo spacing should be kept as short as possible. To achieve a short echo spacing, a non-spatially selective radio-frequency (RF) pulse can be used. Furthermore, to suppress blurring from the $T_{2}$ decay of the late echo, a reduced flip angle of refocusing RF 


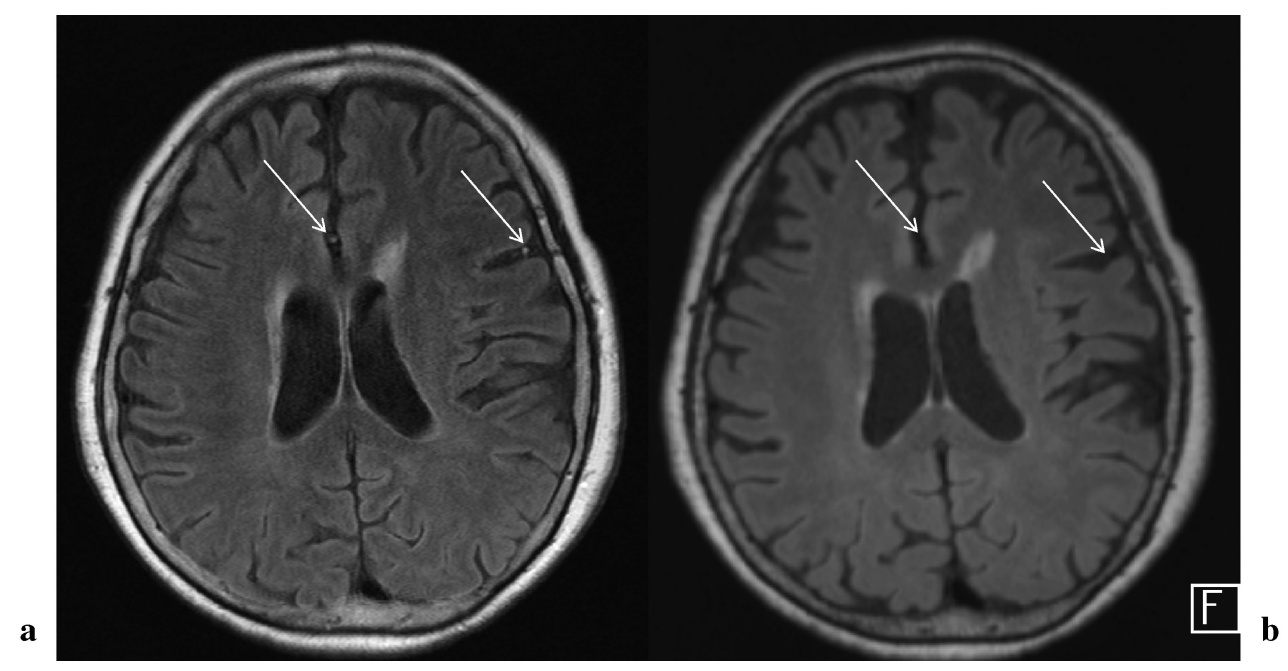

Fig. 2. A 71-year-old woman with cerebral infarction In the 2D-FLAIR image, arterial hyperintensity in the branch of the anterior and middle cerebral arteries with slow flow is visualized (a) (arrows). These high signals are not visible in the 3D-FLAIR image (b) (arrows).

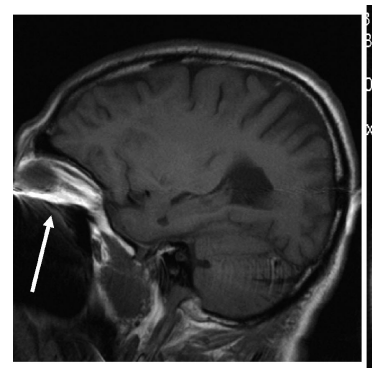

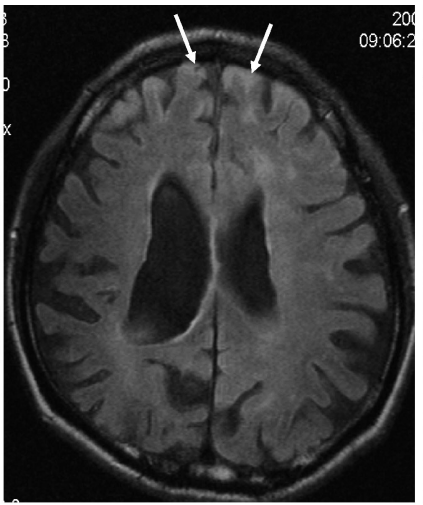

b

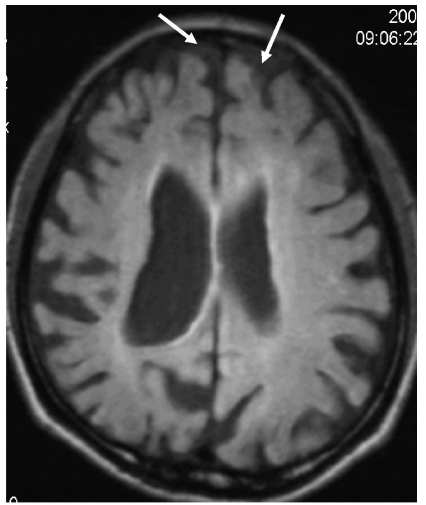

c

Fig. 3. A 62-year-old man with metal dental materials

Severe artifact is seen in the sagittal $\mathrm{T}_{1}$-weighted image (a) (arrow). In the 2DFLAIR image, signal increase of CSF in the frontal subarachnoid space mimics subarachnoid hemorrhage (b) (arrows). This is probably due to incomplete inversion of CSF in the frontal area by the presence of a metallic object. In 3D-FLAIR with non-selective inversion recovery pulse, CSF in the frontal area is properly suppressed (c) (arrows).

pulses should be utilized. ${ }^{15}$ The reduced flip angle in refocusing RF pulses stores the magnetization in the longitudinal direction, thereby avoiding the quick $T_{2}$ decay. This utilizes the fact that the $T_{1}$ relaxation time is far longer than the $T_{2}$ relaxation time. By refocusing the RF pulses with variable flip angles, the stimulated echo contributes to the signals during the echo train. Thus, a very long echo train length can be used in practical terms (Fig. 4). VFA-TSE has vendor-specific names such as SPACE (sampling perfection with application optimized contrasts using different flip-angle evolution; Siemens), Cube (GE), and VISTA (volume isotropic turbo spin echo acquisition; Philips). ${ }^{14,15}$

\section{Flow sensitivity}

The reduced refocusing flip angle of the VFATSE cannot rephase the flowing spins as a $180^{\circ}$ pulse does. The sensitivity to flow is higher in a VFA-TSE than in a conventional TSE. ${ }^{12,14}$ The lack of a vessel signal (usually from velocities over $1 \mathrm{~cm} / \mathrm{s}$ ) in 3 D-FLAIR ${ }^{12}$ makes the recognition of subarachnoid pathologies easier by the suppression of vascular and CSF signals; however, an obscured high-intensity vessel sign in acute stroke cases ${ }^{13}$ or an unclear ivy sign in moyamoya disease ${ }^{12}$ cases 
can be problematic.

\section{Slab-selective excitation}

Although non-selective slab excitation and signal reception by a hard RF pulse allow for reduced echo spacing and increased scan efficiency, some clinical applications such as inner ear imaging need slab-selective excitation to achieve a high resolution while keeping the scan time within a clinically acceptable level. Slab-selective excitation can be performed using an excitation RF pulse that is spatially selective in one or more dimensions. ${ }^{15,17}$ In a VFA-TSE sequence, a relatively long echo spacing is used for the first echo to accommodate the long slab-selective excitation pulse, while a short echo spacing is used for the second and subsequent ech-

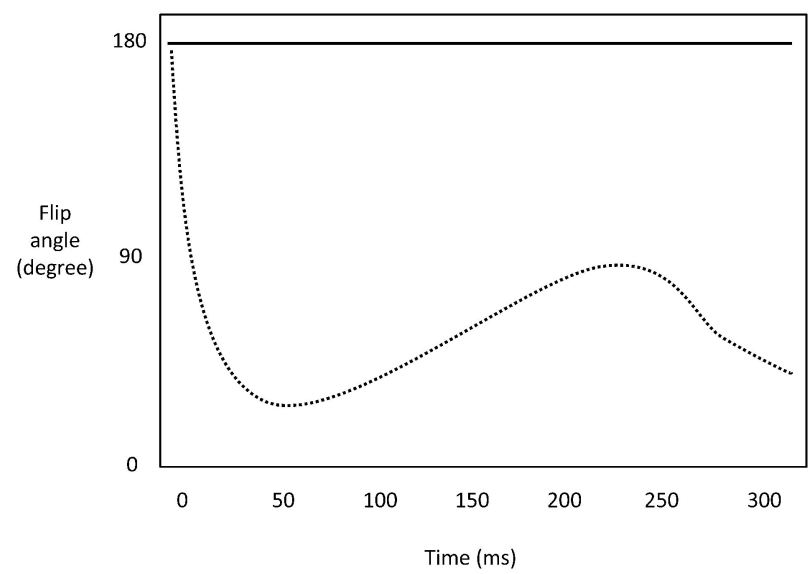

Fig. 4. Schematic plots of flip angles for refocusing RF pulses in conventional turbo spin echo (TSE) and variable flip angle TSE

In conventional TSE, constant $180^{\circ}$ pulses are utilized (straight line). In VFA-TSE, an initial flip angle ramp is followed by less than $180^{\circ}$ (dotted line). This curve is an example. The optimal curve shape of this graph is different among the tissues with different $T_{1}$ and $T_{2}$ values. oes. ${ }^{15}$ To suppress the out-of-slab FID artifacts, partial averaging of more than 1.4 is usually employed. ${ }^{15}$

\section{$T_{2}$-selective inversion recovery}

The scan time of a single-slab 3D-FLAIR tends to be long even with a VFA-TSE, but it can be shortened using the slab-selective excitation described above. In addition, there are other ways to further shorten scan time. Partial Fourier imaging and parallel imaging are routinely applied solutions. Shortening of the repetition time (TR) is another. Usually, a long TR (usually more than $8 \mathrm{~s}$ at $3 \mathrm{~T}$ ) is necessary to sufficiently suppress the signal from the CSF while keeping the adequate signal-tonoise ratio of the brain. ${ }^{18}$ To shorten the TR, $\mathrm{T}_{2}$ selective inversion recovery (IR) has been proposed. In this method, the inversion pulse is replaced with a $90(x)-180(y)-90(x)$ preparation sequence that provides $T_{2}$ selectivity. This allows the magnetization of the tissue with a shorter $\mathrm{T}_{2}$ to recover more rapidly than the CSF with a longer $\mathrm{T}_{2}$, permitting the use of shorter TR values. ${ }^{19}$

The 90(x)-180(y)-90(x) pulses are more susceptible to local magnetic inhomogeneities than regular inversion pulses. The residual fluid signal near the bone and air (such as the labyrinthine fluid) is observed as artifacts (Fig. 5). At 7T, implementation of the FLAIR sequence is difficult as the increased $T_{1}$ weighting from the prolonged $T_{1}$ constants dominates the desired $\mathrm{T}_{2}$ contrast and yields a suboptimal signal-to-noise ratio. Magnetization preparation for 3D-FLAIR has been used at $7 \mathrm{~T}$ to reduce the $T_{1}$ weighting and improve the $T_{2}$ weighting. ${ }^{20}$ There is another way to shorten the TR of a 3D-FLAIR. A shorter TR can be applied in the peripheral part of the $k$-space while smoothly modulating TR and TI throughout the $k$-space. Scan time can be reduced effectively by this approach,

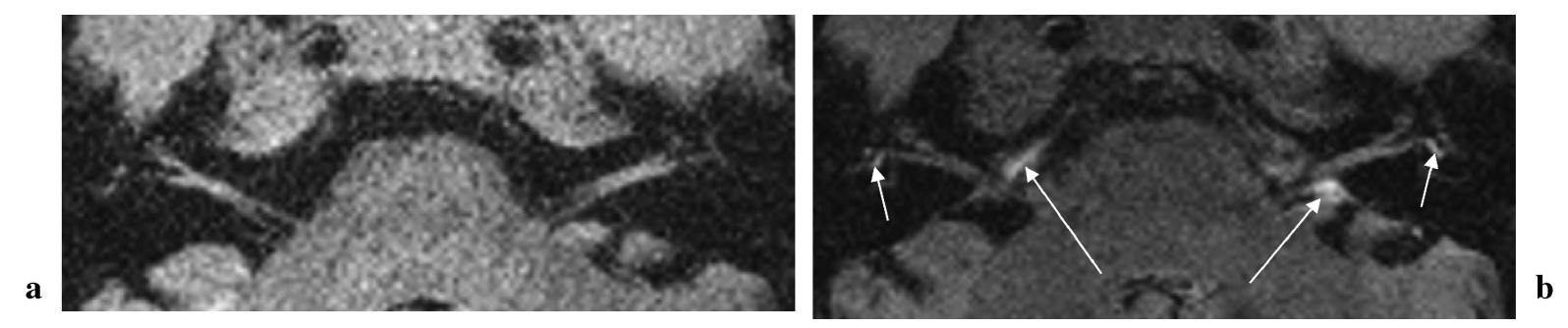

Fig. 5. A 3D-FLAIR image by conventional inversion recovery pulse (a) and that by $\mathrm{T}_{2}$-selective inversion recovery pulse (b) at $3 \mathrm{~T}$

The repetition time is $9,000 \mathrm{~ms}$ for (a) and 5,000 $\mathrm{ms}$ for (b). The fluid shows a high signal in (b) at the area near the air or bone due to an imperfect inversion by field inhomogeneity (arrows). Long arrows indicate the cerebellopontine angle cistern, and short arrows indicate the lateral part of the vestibule. $\mathrm{A} \mathrm{T}_{2}$-selective inversion recovery pulse is more susceptible to local magnetic field inhomogeneities than a regular inversion recovery pulse. 


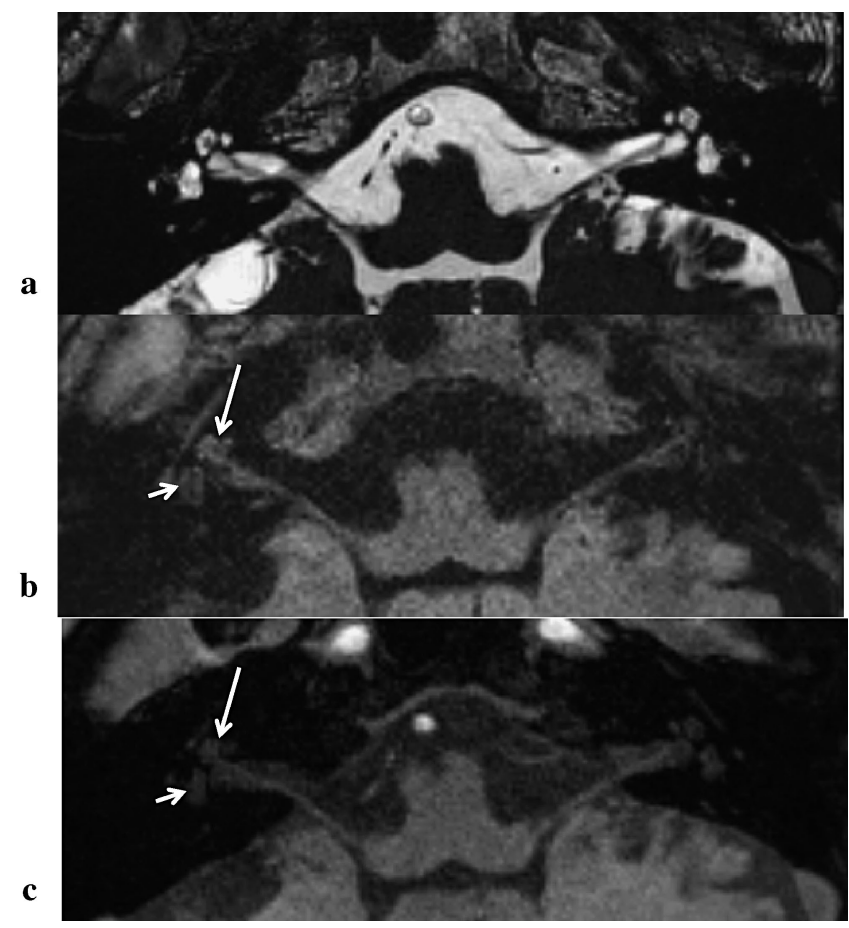

Fig. 6. A 60-year-old man with sudden deafness in the right ear

(a) CISS image, (b) non-contrast-enhanced 3DFLAIR, and (c) non-contrast-enhanced $\mathrm{T}_{1}$-weighted image. Lymph fluid in the right cochlea (long arrow) and right vestibule (short arrow) show increased signal on 3D-FLAIR imaging compared to the other side. This is presumed to be due to an increased protein concentration or slight hemorrhage in the right labyrinth. A signal increase in the right labyrinth cannot be detected on the $\mathrm{T}_{1}$-weighted image.

while keeping the contrast-to-noise ratio similar to a constant TR sequence. ${ }^{18}$

\section{High sensitivity to subtle $T_{1}$ changes in fluid}

FLAIR is reported to be more sensitive to subtle $\mathrm{T}_{1}$ changes in fluid than conventional $\mathrm{T}_{1}$-weighted images. ${ }^{21-30}$ A FLAIR image allows the detection of subtle subarachnoid hemorrhage in the CSF space, ${ }^{9}$ signal increases in CSF after the inhalation of oxygen, ${ }^{31}$ and subtle compositional changes of the labyrinthine fluid (Figs. 6 and 7), 26,27,30,32-34 which can be difficult to recognize on $\mathrm{T}_{1}$-weighted images. Heavily $\mathrm{T}_{2}$-weighted 3D-FLAIR has been reported to be much more sensitive than regularcontrast 3D-FLAIR. ${ }^{35}$ After intravenous administration of a single dose of gadolinium-based contrast media (IV-SD-GBCM), a signal increase in various fluid spaces such as the anterior eye segment, optic nerve sheath, Meckel's cave, ambient cistern, internal auditory canal, and perilymph of the labyrinth has been reported. ${ }^{36-39}$

\section{Prospective motion correction}

The scan time for a 3D-FLAIR is usually several minutes to $15 \mathrm{~min}$. Patient motion during the scan results in blurring of the images. Fortunately, there are "dead" periods without RF pulses and gradient activities while waiting for the signal recovery during the long TR of a single-slab 3D-FLAIR. Thus, there is enough time to insert an EPI-based navigator scan at every TR. This approach can update the position and orientation of the slab and avoid the spin history effect that cannot be eliminated by retrospective motion correction. ${ }^{40}$

\section{Local excitation by parallel transmission}

An advanced slab-selective excitation technique has been introduced recently. Inner-volume imaging using a three-dimensional parallel spatially selective excitation allows for reduced field-of-view imaging of selectively excited targets. This produces a higher spatial resolution and significantly reduced measurement times in vivo. This approach is useful for a reduction of the distortion in EPI-based diffusion-weighted images, as well as a reduction in the blurring artifacts on VFA-TSE. ${ }^{41}$

\section{Clinical Applications}

\section{Multiple sclerosis}

FLAIR is useful for the evaluation of pathologies near or in the fluid space. For 3D-FLAIR, there are many reports regarding application to multiple sclerosis. ${ }^{5,6,42-54}$ Smaller lesions can be detected by 3D-FLAIR than by 2D-FLAIR due to thinner slices and fewer flow artifacts with 3D-FLAIR. ${ }^{45,46} \mathrm{Ex}$ cellent detection of cortical lesions by 3D-FLAIR has also been reported (Fig. 8). ${ }^{43,44}$ Cortical lesions are rather specific for multiple sclerosis (MS), and they occur in the earliest stages of the disease. ${ }^{50} \mathrm{It}$ is clinically important to make cortical lesions visible in vivo by clinical 3D MR imaging. At both 1.5 and $3 \mathrm{~T},{ }^{48,55}$ double inversion recovery (DIR) sequence is more sensitive in detecting cortical lesions than 3D-FLAIR; however, 3D-FLAIR is reported to be more sensitive than DIR at $7 \mathrm{~T} .{ }^{50}$ In the present clinical setting, 3D-FLAIR is one of the important multi-contrast pulse sequences to evaluate cortical lesions. Computer-based quantitative evaluation can be applied to 3D-FLAIR by the reduction of partial volume averaging effects. ${ }^{5,6} \mathrm{MS}$ lesions usually follow a perivascular orientation. Typical MS lesions run along the veins that stretch from the lateral ventricles into the brain. ${ }^{50}$ Perivascular lesion orientation can be appreciated on 3DFLAIR for MS lesions by combining with $\mathrm{T}_{2}{ }^{*}$ weighted imaging. ${ }^{50}$ A combination of 3 D-FLAIR 


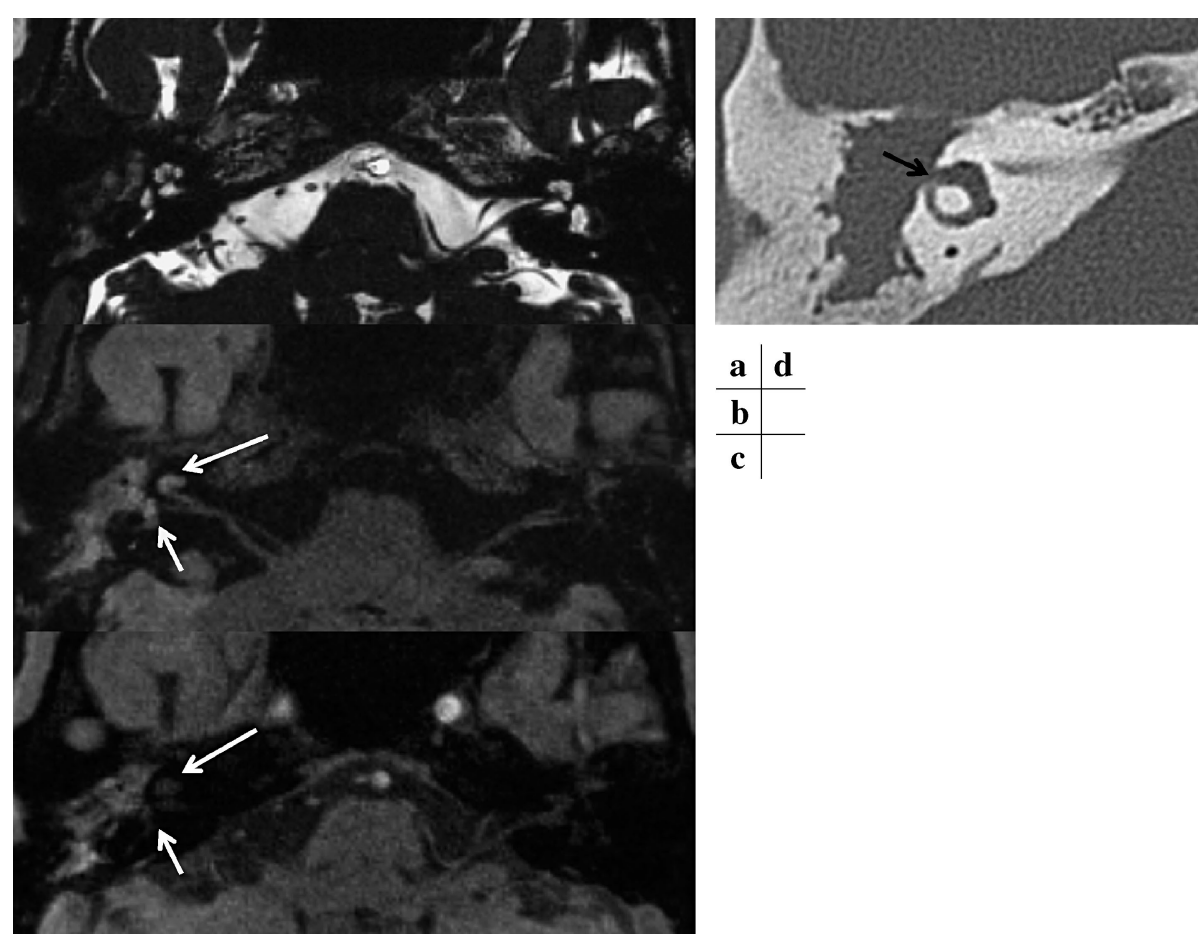

Fig. 7. A 43-year-old man with a right labyrinthine fistula from a cholesteatoma (a) CISS image, (b) non-contrast-enhanced 3D-FLAIR, (c) non-contrast-enhanced $\mathrm{T}_{1}$-weighted image, and (d) $\mathrm{CT}$ image of the right side. Lymph fluid in the right cochlea (long arrow) and right vestibule (short arrow) shows an increased signal on 3D-FLAIR imaging compared to the other side. This is presumed to be due to an increased protein concentration or slight hemorrhage in the right labyrinth by invasion of a cholesteatoma. A signal increase in the right labyrinth cannot be detected on the $\mathrm{T}_{1}$-weighted image. (d) A fistula to the lateral semicircular canal from a cholesteatoma is visualized (black arrow).

and susceptibility-weighted imaging (SWI) or $\mathrm{T}_{2}{ }^{*}$ weighted imaging is useful for the assessment of perivascular lesions. ${ }^{50,56}$

\section{Cerebrovascular disease}

3D-FLAIR allows FLAIR imaging with virtually no CSF artifacts and is, thus, particularly useful for SAH detection (Fig. 9). ${ }^{9}$ Lack of visualization for intra-arterial signal in the patients with acute ischemic stroke in 3D-FLAIR is one of the drawbacks. ${ }^{13}$

Cortical microinfarcts (CMIs) are detected as small foci restricted to the cerebral cortex in autopsy brains. CMIs are thought to be caused by cerebral amyloid angiopathy in the elderly and may be a risk for dementia. It has been reported that CMIs can be detected by 3D-FLAIR and DIR at $3 \mathrm{~T}^{57}$ High-signal-intensity abnormalities within the drainage territory of developmental venous anomalies were also observed on 3D-FLAIR around vascular structures. ${ }^{56}$

\section{Meningeal pathologies}

Due to a higher sensitivity to subtle compositional changes from fluid and a reduced incidence of artifacts from CSF motion, post-contrast 3DFLAIR is valuable for the evaluation of various kinds of meningitis. ${ }^{21,58,59}$ On post-contrast 3DFLAIR images, enhancement in the fundus of the internal auditory canal (IAC) was frequently observed in patients with meningeal disease, even at 10 min after administration of the contrast agent. This enhancement in the IAC fundus is not discernable on $\mathrm{T}_{1}$-weighted $3 \mathrm{D}$-FLASH images. ${ }^{58}$ The rim patterns of intracranial meningiomas on non-enhanced 3D-FLAIR images were reported to be useful to predict surgical cleavability and for histological tumor grading. ${ }^{60}$

\section{Visualization of white matter tracts}

For the visualization of neuronal white matter tracts, diffusion tensor imaging (DTI) is widely used. DTI is usually performed with single-shot echo planar imaging, which is susceptible to distortion. On non-contrast-enhanced 3D-FLAIR, the brainstem tracts can be delineated and correlated with DTI results. ${ }^{61}$ More conspicuous tract visualization has been reported by non-contrast-enhanced heavily $\mathrm{T}_{2}$-weighted 3D-FLAIR. Distortion-free, 
volumetric image presentation of tracts is also possible by non-contrast-enhanced heavily $\mathrm{T}_{2}$-weighted 3D-FLAIR (Fig. 10). ${ }^{62}$

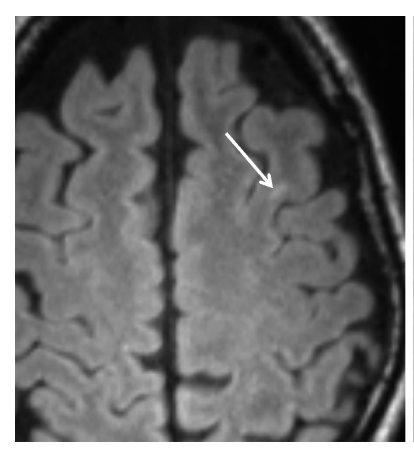

a

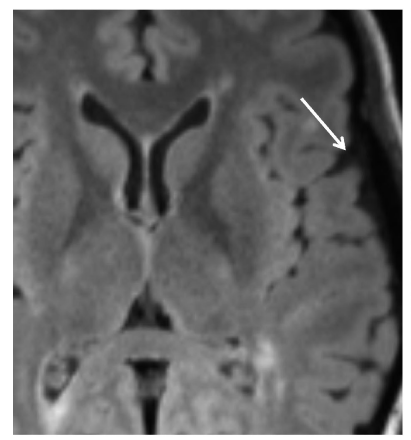

c

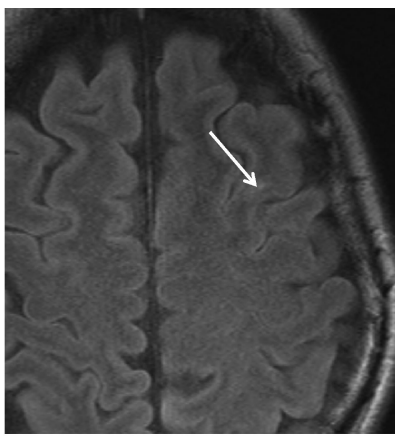

b

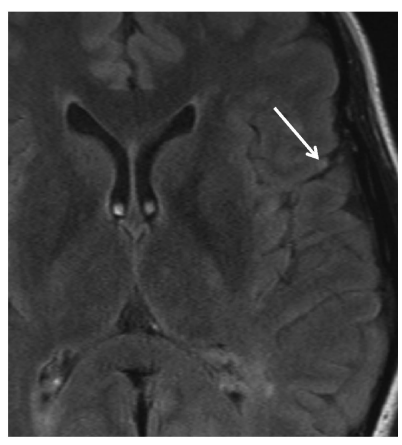

d
Fig. 8. A 38-year-old woman with multiple sclerosis A cortical lesion in the left frontal lobe cortex is visualized in the 1-mm-thick 3D-FLAIR image (a) (arrow), but the precise location of this lesion is unclear in the 4.5-mm-thick 2D-FLAIR image (b) (arrow). In the lower level slice, no cortical lesion is seen around the Sylvian fissure in the 1-mm-thick 3D-FLAIR image (c) (arrow); however, a high-signal artifact in CSF mimics a cortical lesion in the 4.5-mm-thick 2DFLAIR image (d) (arrow).

\section{Signal change in the labyrinthine fluid}

\section{Sudden deafness}

In ears with sudden deafness, labyrinthine fluid signal increase is frequently observed on $3 \mathrm{D}$ FLAIR, but not on $\mathrm{T}_{1}$-weighted $3 \mathrm{D}$ gradient echo images. ${ }^{32,63}$ An increased contrast enhancement of the labyrinthine fluid on 3D-FLAIR is sometimes observed in ears with sudden deafness after an intravenous administration of a gadolinium-based contrast material. ${ }^{32,64}$ This contrast enhancement suggests the breakdown of the blood-labyrinthine barrier. ${ }^{63}$ Prognosis for hearing was not good in ears with a high signal on precontrast 3DFLAIR. ${ }^{65,66}$

\section{Inflammation}

A signal increase in the labyrinthine fluid on 3DFLAIR in various inflammatory diseases such as acute meningitis, acute otitis media, Wegener's granulomatosis, ${ }^{27}$ mumps deafness, ${ }^{26}$ RamsayHunt syndrome ${ }^{67-69}$ and labyrinthine fistula by a cholesteatoma ${ }^{70}$ has been reported. Signal increase in the labyrinthine fluid on precontrast 3D-FLAIR suggests the presence of a small degree of hemorrhage or protein, and on post-contrast $3 \mathrm{D}$-FLAIR, enhancement of the labyrinthine fluid suggests the active breakdown of the blood-labyrinthine barrier. $^{27,30}$

\section{Vestibular schwannoma}

A signal change of the labyrinthine fluid on 3DFLAIR was also observed in ears with vestibular schwannoma. ${ }^{71}$ The results of this study suggested that an alteration of cochlear fluid composition and an increased permeability of the blood-labyrinthine barrier existed in the affected side in patients with vestibular schwannoma. Furthermore, although weak, a positive correlation between the post-con-
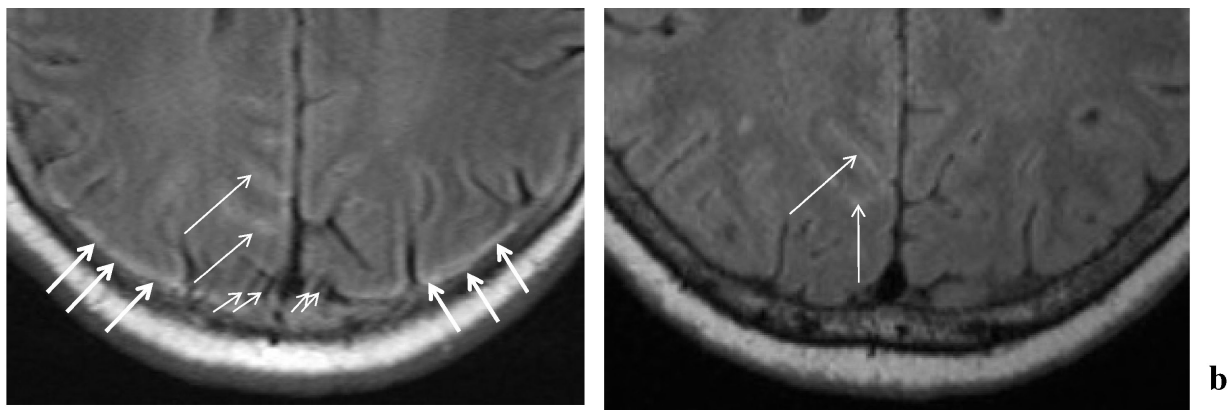

Fig. 9. A 56-year-old woman with hypertension and severe headache Subtle subarachnoid hemorrhage in the sulcus is unclear in the 5-mm-thick 2DFLAIR image (a) (thin arrows) but can be recognized on the 1-mm-thick 3DFLAIR image (b) (thin arrows). Note that ghost artifact from the superior sagittal sinus (a) (short arrows) and chemical shift artifact in the slice-select direction by fatty marrow of the inner table (a) (bold arrows) overlap the surface of the brain. These artifacts in 2D-FLAIR make the recognition of subtle subarachnoid hemorrhage further difficult. 

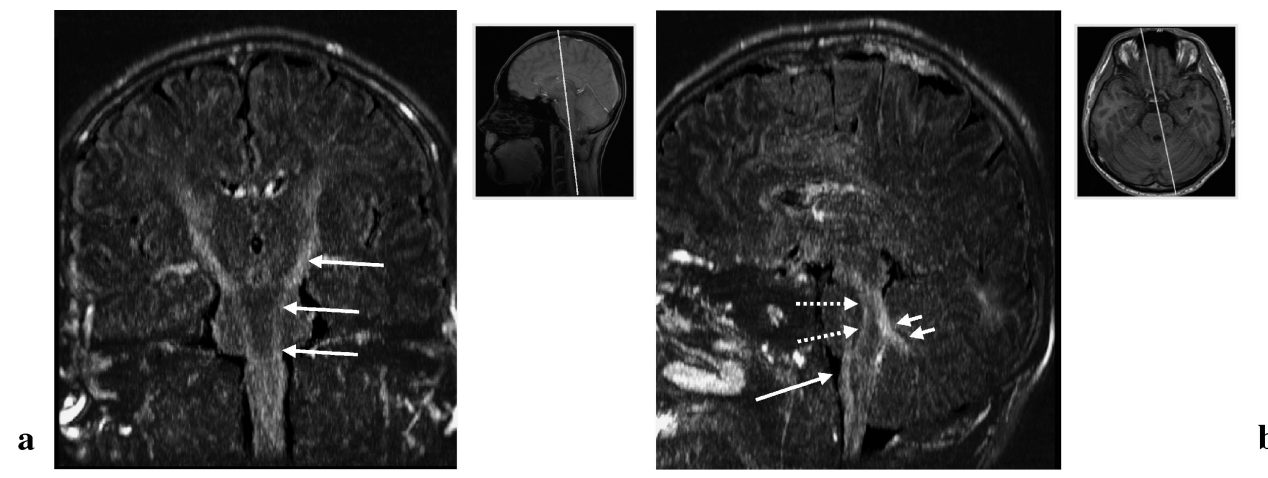

Fig. 10. Non-diffusion-based tractography

A thin-slab maximum intensity projection image $\left(5 \mathrm{~mm}\right.$ thick) of a heavily $\mathrm{T}_{2}$ weighted 3D-FLAIR image of the brain in a healthy subject. (a) Coronal projection image. The corticospinal tract (arrows) is visualized on both sides. (b) Oblique sagittal projection image. The corticospinal tract (arrow), medial lemniscus (dotted arrows), and superior cerebellar peduncle (short arrows) are clearly visualized. Note that there is no distortion artifact as seen on EPI-based DTI tractography.

trast cochlear signal intensity on 3D-FLAIR and hearing level was observed. The cochlear signal on 3D-FLAIR may be useful as an additional parameter when monitoring the degree of functional impairment during follow-up of patients with a small vestibular schwannoma confined to the internal auditory canals. ${ }^{72}$ Incidentally, endolymphatic hydrops can be recognized on non-contrast-enhanced 3D-FLAIR images due to the signal increase of the perilymph. ${ }^{33}$

\section{Endolymphatic hydrops}

Visualization of endolymphatic hydrops (EH) in patients with Ménière's disease was first achieved using 3D-FLAIR obtained at $24 \mathrm{~h}$ after intratympanic administration of GBCM (IT-GBCM) ${ }^{25}$ Enhancement of the perilymph was not found on $\mathrm{T}_{1}$-weighted $3 \mathrm{D}$ gradient echo images. Numerous reports have been published using ITGBCM. ${ }^{24,29,73-95}$ The IT-GBCM method opens the possibility for an objective diagnosis of Ménière's disease. The relationship between EH and symptoms is under investigation. However, there are some drawbacks to the IT-GBCM method. One is that the IT administration is an off-label use of GBCM. A second drawback is that the GBCM penetration of the round window membrane is insufficient to visualize EH in nearly $20 \%$ of patients. ${ }^{89}$ Third, $24 \mathrm{~h}$ of waiting time is needed after the administration of GBCM before starting the MR imaging. ${ }^{24}$ The final drawback is that only the injected ear can be evaluated. One advantage of ITGBCM is the capability for simulation of the intralabyrinthine distribution of the intratympanically administered drug. ${ }^{96}$ Although IT-GBCM was usu- ally performed through the tympanic membrane, some researchers administered the GBCM through the Eustachian tube. ${ }^{97}$

To decrease the invasiveness of IT-GBCM, evaluation of $\mathrm{EH}$ by intravenous administration of GBCM (IV-GBCM) was tried. A combination of double-dose IV-GBCM and 3D-FLAIR imaging enabled the visualization of EH. ${ }^{98-100}$ Usually, $4 \mathrm{~h}$ after IV-GBCM, MR imaging can be conducted.

To further decrease the invasiveness, a single dose of IV-GBCM and heavily $\mathrm{T}_{2}$-weighted $3 \mathrm{D}$ FLAIR enabled the visualization of $\mathrm{EH}^{39}$ By the development of a single-dose IV-GBCM method, the imaging evaluation of $\mathrm{EH}$ became clinically feasible, and various studies using this method have been reported (Fig. 11). ${ }^{37,38,101-108}$

In summary, 3D-FLAIR using VFA-TSE has several unique advantages for clinical neuroimaging as shown below:

1) Thinner slices and multi-planar reformation capability,

2) Higher flow sensitivity,

3) High sensitivity to subtle $T_{1}$ changes in fluid,

4) Free from CSF inflow artifacts,

5) Reasonable scan time, and

6) A 3D dataset that allows computer-aided analysis.

Familiarity with these features of 3D-FLAIR imaging and proper use of 3D-FLAIR are important in order for radiologists to obtain useful information for patient management.

\section{Acknowledgements}

This review was partly supported by Grants-in- 

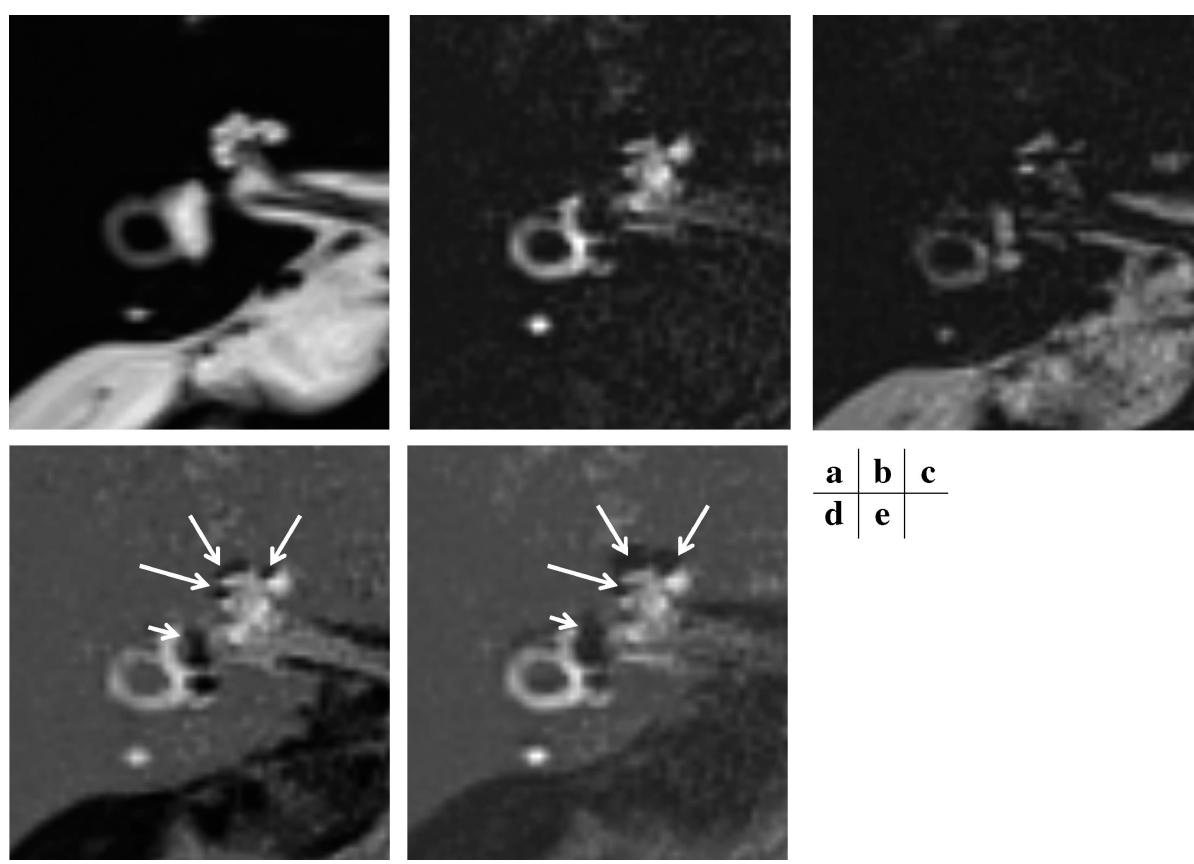

Fig. 11. A 73-year-old man with right Ménière's disease

(a) An MR cisternographic image (MRC) and (b) a heavily $\mathrm{T}_{2}$-weighted 3DFLAIR image obtained $4 \mathrm{~h}$ after intravenous administration of a single dose of a gadolinium-based contrast material. The perilymph shows high signal in this positive perilymph image (PPI). (c) A positive endolymph image (PEI) obtained by a shortening of the inversion time of the PPI. (d) The subtraction image of the PEI from the PPI (HYbriD of Reversed image Of Positive endolymph signal and native image of positive perilymph Signal, HYDROPS) provides an image that can be easily interpreted. The long arrows indicate a significantly dilated endolymphatic space in the right cochlea. The short arrow indicates a significantly dilated vestibular endolymphatic space. (e) Subtraction of the MRC from the PPI provides a similar contrast appearance image as (d). This image is called a HYDROPS2 image. A HYDROPS2 (HYbriD of Reversed image Of MR cisternography and positive Perilymph Signal by heavily $\mathrm{T}_{2}$-weighted 3D-FLAIR) image can be obtained in a shorter scan time than a HYDROPS image.

Aid for Scientific Research from the Japan Society for the Promotion of Science (JSPS KAKENHI, numbers 25293263 and 24659562) and by a Grantin-Aid for Research on Intractable Vestibular Disorder from the Ministry of Health, Labour and Welfare of Japan to SN.

\section{References}

1. Nakajima R, Uchino A, Saito N, Nishikawa R. Ring-shaped lateral ventricular nodules detected with brain MR imaging. Magn Reson Med Sci 2013; 12:105-110.

2. Rydberg JN, Hammond CA, Grimm RC, et al. Initial clinical experience in MR imaging of the brain with a fast fluid-attenuated inversion-recovery pulse sequence. Radiology 1994; 193:173-180.

3. Naganawa S, Koshikawa T, Nakamura T, et al. Comparison of flow artifacts between 2D-FLAIR and 3D-FLAIR sequences at 3T. Eur Radiol 2004;
14:1901-1908.

4. Kallmes DF, Hui FK, Mugler JP 3rd. Suppression of cerebrospinal fluid and blood flow artifacts in FLAIR MR imaging with a single-slab three-dimensional pulse sequence: initial experience. Radiology 2001; 221:251-255.

5. Tan IL, van Schijndel RA, Pouwels PJ, Ader HJ, Barkhof F. Serial isotropic three-dimensional fast FLAIR imaging: using image registration and subtraction to reveal active multiple sclerosis lesions. AJR Am J Roentgenol 2002; 179:777-782.

6. Bilello M, Arkuszewski M, Nasrallah I, Wu X, Erus G, Krejza J. Multiple sclerosis lesions in the brain: computer-assisted assessment of lesion load dynamics on 3D FLAIR MR images. Neuroradiol J 2012; 25:17-21.

7. De Coene B, Hajnal JV, Gatehouse P, et al. MR of the brain using fluid-attenuated inversion recovery (FLAIR) pulse sequences. AJNR Am J Neuroradiol 1992; 13:1555-1564.

8. Korogi Y, Sugahara T, Shigematsu Y, et al. Ultrafast 
FLAIR imaging with single-shot echo-planar technique in evaluation of intracranial lesions. Comput Med Imaging Graph 1999; 23:119-126.

9. Lummel N, Schoepf V, Burke M, Brueckmann H, Linn J. 3D fluid-attenuated inversion recovery imaging: reduced CSF artifacts and enhanced sensitivity and specificity for subarachnoid hemorrhage. AJNR Am J Neuroradiol 2011; 32:20542060.

10. Ozcan UA, Isik U, Ozpinar A, Baykan N, Dincer A. Assessment of sedated pediatric brain with 3DFLAIR sequence at 3T MRI. Brain Dev 2014; pii: S0387-7604(0314)00211-00213.

11. Barker GJ. 3D fast FLAIR: a CSF-nulled 3D fast spin-echo pulse sequence. Magn Reson Imaging 1998; 16:715-720.

12. Kakeda S, Korogi Y, Hiai Y, Ohnari N, Sato T, Hirai T. Pitfalls of 3D FLAIR brain imaging: a prospective comparison with 2D FLAIR. Acad Radiol 2012; 19:1225-1232.

13. Hodel J, Leclerc X, Rodallec M, et al. Fluid-attenuated inversion recovery vascular hyperintensities are not visible using 3D CUBE FLAIR sequence. Eur Radiol 2013; 23:1963-1969.

14. Naganawa S, Kawai H, Fukatsu H, et al. Highspeed imaging at 3 Tesla: a technical and clinical review with an emphasis on whole-brain $3 \mathrm{D}$ imaging. Magn Reson Med Sci 2004; 3:177-187.

15. Mugler JP 3rd. Optimized three-dimensional fastspin-echo MRI. J Magn Reson Imaging 2014; 39: 745-767.

16. Lichy MP, Wietek BM, Mugler JP 3rd, et al. Magnetic resonance imaging of the body trunk using a single-slab, 3-dimensional, $\mathrm{T}_{2}$-weighted turbospin-echo sequence with high sampling efficiency (SPACE) for high spatial resolution imaging: initial clinical experiences. Invest Radiol 2005; 40:754760.

17. Park J, Mugler JP 3rd, Hughes T. Reduction of B1 sensitivity in selective single-slab 3D turbo spin echo imaging with very long echo trains. Magn Reson Med 2009; 62:1060-1066.

18. Gai ND, Butman JA. Reduced scan time three-dimensional FLAIR using modulated inversion and repetition time. J Magn Reson Imaging 2014 Jun 30. [Epub ahead of print]

19. Wong EC, Liu TT, Luh WM, Frank LR, Buxton RB. $T(1)$ and $T(2)$ selective method for improved SNR in CSF-attenuated imaging: T(2)-FLAIR. Magn Reson Med 2001; 45:529-532.

20. Visser F, Zwanenburg JJ, Hoogduin JM, Luijten PR. High-resolution magnetization-prepared 3DFLAIR imaging at 7.0 Tesla. Magn Reson Med 2010; 64:194-202.

21. Hirai T, Ando Y, Yamura M, et al. Transthyretinrelated familial amyloid polyneuropathy: evaluation of CSF enhancement on serial $\mathrm{T}_{1}$-weighted and fluid-attenuated inversion recovery images following intravenous contrast administration. AJNR
Am J Neuroradiol 2005; 26:2043-2048.

22. Lee IH, Kim HJ, Chung WH, et al. Signal intensity change of the labyrinth in patients with surgically confirmed or radiologically diagnosed vestibular schwannoma on isotropic 3D fluid-attenuated inversion recovery MR imaging at 3T. Eur Radiol 2010; 20:949-957.

23. Naganawa S, Nakashima T. Cutting edge of inner ear MRI. Acta Otolaryngol Suppl 2009:15-21.

24. Nakashima T, Naganawa S, Katayama N, et al. Clinical significance of endolymphatic imaging after intratympanic gadolinium injection. Acta Otolaryngol Suppl 2009:9-14.

25. Nakashima T, Naganawa S, Sugiura M, et al. Visualization of endolymphatic hydrops in patients with Meniere's disease. Laryngoscope 2007; 117:415420.

26. Otake H, Sugiura M, Naganawa S, Nakashima T. 3D-FLAIR magnetic resonance imaging in the evaluation of mumps deafness. Int J Pediatr Otorhinolaryngol 2006; 70:2115-2117.

27. Sone M, Mizuno T, Naganawa S, Nakashima T. Imaging analysis in cases with inflammation-induced sensorineural hearing loss. Acta Otolaryngol 2009; 129:239-243.

28. Sugiura M, Naganawa S, Nakashima T, Misawa H, Nakamura T. Magnetic resonance imaging of endolymphatic sac in acute low-tone sensorineural hearing loss without vertigo. ORL J Otorhinolaryngol Relat Spec 2003; 65:254-260.

29. Yamazaki M, Naganawa S, Kawai H, Nihashi T, Nakashima T. Signal alteration of the cochlear perilymph on 3 different sequences after intratympanic Gd-DTPA administration at 3 tesla: comparison of

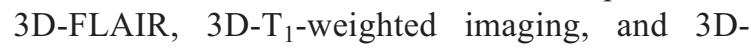
CISS. Magn Reson Med Sci 2010; 9:65-71.

30. Naganawa S, Ishihara S, Iwano S, Sone $M$, Nakashima T. Detection of presumed hemorrhage in the ampullar endolymph of the semicircular canal: a case report. Magn Reson Med Sci 2009; 8: 187-191.

31. Mehemed TM, Fushimi Y, Okada T, et al. Dynamic oxygen-enhanced MRI of cerebrospinal fluid. PLoS One 2014; 9:e100723.

32. Sugiura M, Naganawa S, Teranishi M, Nakashima $\mathrm{T}$. Three-dimensional fluid-attenuated inversion recovery magnetic resonance imaging findings in patients with sudden sensorineural hearing loss. Laryngoscope 2006; 116:1451-1454.

33. Naganawa S, Kawai H, Sone M, Nakashima T, Ikeda M. Endolympathic hydrops in patients with vestibular schwannoma: visualization by non-contrast-enhanced 3D FLAIR. Neuroradiology 2011; 53:1009-1015.

34. Naganawa S, Sone M, Otake H, Nakashima T. Endolymphatic hydrops of the labyrinth visualized on noncontrast MR imaging: a case report. Magn Reson Med Sci 2009; 8:43-46.

35. Naganawa S, Kawai H, Sone M, Nakashima T. In- 
creased sensitivity to low concentration gadolinium contrast by optimized heavily $\mathrm{T}_{2}$-weighted $3 \mathrm{D}$ FLAIR to visualize endolymphatic space. Magn Reson Med Sci 2010; 9:73-80.

36. Naganawa S, Suzuki K, Yamazaki M, Sakurai Y. Serial scans in healthy volunteers following intravenous administration of gadoteridol: time course of contrast enhancement in various cranial fluid spaces. Magn Reson Med Sci 2014; 13:7-13.

37. Naganawa S, Yamazaki M, Kawai H, Bokura K, Sone M, Nakashima T. Visualization of endolymphatic hydrops in Ménière's disease after intravenous administration of single-dose gadodiamide at 1.5T. Magn Reson Med Sci 2013; 12:137-139.

38. Naganawa S, Yamazaki M, Kawai H, Bokura K, Sone M, Nakashima T. Visualization of endolymphatic hydrops in Ménière's disease after singledose intravenous gadolinium-based contrast medium: timing of optimal enhancement. Magn Reson Med Sci 2012; 11:43-51.

39. Naganawa S, Yamazaki M, Kawai H, Bokura K, Sone M, Nakashima T. Visualization of endolymphatic hydrops in Ménière's disease with singledose intravenous gadolinium-based contrast media using heavily $\mathrm{T}_{2}$-weighted 3D-FLAIR. Magn Reson Med Sci 2010; 9:237-242.

40. Tisdall MD, Hess AT, Reuter M, Meintjes EM, Fischl B, van der Kouwe AJ. Volumetric navigators for prospective motion correction and selective reacquisition in neuroanatomical MRI. Magn Reson Med 2012; 68:389-399.

41. Schneider JT, Kalayciyan R, Haas M, et al. Innervolume imaging in vivo using three-dimensional parallel spatially selective excitation. Magn Reson Med 2013; 69:1367-1378.

42. Simoes R, Monninghoff C, Dlugaj M, et al. Automatic segmentation of cerebral white matter hyperintensities using only 3D FLAIR images. Magn Reson Imaging 2013; 31:1182-1189.

43. Mike A, Glanz BI, Hildenbrand P, et al. Identification and clinical impact of multiple sclerosis cortical lesions as assessed by routine 3T MR imaging. AJNR Am J Neuroradiol 2011; 32:515-521.

44. Kilsdonk ID, de Graaf WL, Soriano AL, et al. Multicontrast MR imaging at $7 \mathrm{~T}$ in multiple sclerosis: highest lesion detection in cortical gray matter with 3D-FLAIR. AJNR Am J Neuroradiol 2013; 34: 791-796.

45. Bink A, Schmitt M, Gaa J, Mugler JP 3rd, Lanfermann H, Zanella FE. Detection of lesions in multiple sclerosis by 2D FLAIR and single-slab 3D FLAIR sequences at 3.0T: initial results. Eur Radiol 2006; 16:1104-1110.

46. Paniagua Bravo A, Sanchez Hernandez JJ, Ibanez Sanz L, Alba de Caceres I, Crespo San Jose JL, Garcia-Castano Gandariaga B. A comparative MRI study for white matter hyperintensities detection: 2D-FLAIR, FSE PD 2D, 3D-FLAIR and FLAIR MIP. Br J Radiol 2014; 87:20130360.
47. Polak P, Magnano C, Zivadinov R, Poloni G. 3D FLAIRED: 3D fluid attenuated inversion recovery for enhanced detection of lesions in multiple sclerosis. Magn Reson Med 2012; 68:874-881.

48. Moraal B, Roosendaal SD, Pouwels PJ, et al. Multicontrast, isotropic, single-slab 3D MR imaging in multiple sclerosis. Eur Radiol 2008; 18:2311-2320.

49. Jurcoane A, Wagner M, Schmidt C, et al. Withinlesion differences in quantitative MRI parameters predict contrast enhancement in multiple sclerosis. J Magn Reson Imaging 2013; 38:1454-1461.

50. Kilsdonk ID, Wattjes MP, Lopez-Soriano A, et al. Improved differentiation between MS and vascular brain lesions using FLAIR* at 7 Tesla. Eur Radiol 2014; 24:841-849.

51. Geurts JJ, Pouwels PJ, Uitdehaag BM, Polman CH, Barkhof F, Castelijns JA. Intracortical lesions in multiple sclerosis: improved detection with 3D double inversion-recovery MR imaging. Radiology 2005; 236:254-260.

52. Kober T, Granziera C, Ribes D, et al. MP2RAGE multiple sclerosis magnetic resonance imaging at 3T. Invest Radiol 2012; 47:346-352.

53. Seewann A, Kooi EJ, Roosendaal SD, et al. Postmortem verification of MS cortical lesion detection with 3D DIR. Neurology 2012; 78:302-308.

54. Gramsch C, Nensa F, Kastrup O, et al. Diagnostic value of 3D fluid attenuated inversion recovery sequence in multiple sclerosis. Acta Radiol 2014; pii: 0284185114534413. [Epub ahead of print]

55. Wattjes MP, Lutterbey GG, Gieseke J, et al. Double inversion recovery brain imaging at $3 \mathrm{~T}$ : diagnostic value in the detection of multiple sclerosis lesions. AJNR Am J Neuroradiol 2007; 28:54-59.

56. Umino M, Maeda M, Matsushima N, Matsuura K, Yamada T, Sakuma H. High-signal-intensity abnormalities evaluated by 3D fluid-attenuated inversion recovery imaging within the drainage territory of developmental venous anomalies identified by susceptibility-weighted imaging at 3T. Jpn J Radiol 2014; 32:397-404.

57. Ii Y, Maeda M, Kida H, et al. In vivo detection of cortical microinfarcts on ultrahigh-field MRI. J Neuroimaging 2013; 23:28-32.

58. Naganawa S, Sugiura M, Kawamura M, Fukatsu H, Nakashima T, Maruyama K. Prompt contrast enhancement of cerebrospinal fluid space in the fundus of the internal auditory canal: observations in patients with meningeal diseases on 3D-FLAIR images at 3 Tesla. Magn Reson Med Sci 2006; 5:151155.

59. Fukuoka H, Hirai T, Okuda T, et al. Comparison of the added value of contrast-enhanced 3D fluid-attenuated inversion recovery and magnetization-prepared rapid acquisition of gradient echo sequences in relation to conventional postcontrast $\mathrm{T}_{1}$-weighted images for the evaluation of leptomeningeal diseases at 3T. AJNR Am J Neuroradiol 2010; 31:868873. 
60. Enokizono M, Morikawa M, Matsuo T, et al. The rim pattern of meningioma on 3D FLAIR imaging: correlation with tumor-brain adhesion and histological grading. Magn Reson Med Sci 2014; 13:251260.

61. Kitajima M, Hirai T, Shigematsu Y, et al. Comparison of 3D FLAIR, 2D FLAIR, and 2D $\mathrm{T}_{2}$-weighted MR imaging of brain stem anatomy. AJNR Am J Neuroradiol 2012; 33:922-927.

62. Yamazaki M, Naganawa S, Kawai H, et al. Visualization of white matter tracts using a non-diffusion weighted magnetic resonance imaging method: does intravenous gadolinium injection four hours prior to the examination affect the visualization of white matter tracts? PLoS One 2014; 9:e91860.

63. Berrettini S, Seccia V, Fortunato S, et al. Analysis of the 3-dimensional fluid-attenuated inversion-recovery (3D-FLAIR) sequence in idiopathic sudden sensorineural hearing loss. JAMA Otolaryngol Head Neck Surg. 2013; 139:456-464.

64. Zhu H, Ou Y, Fu J, Zhang Y, Xiong H, Xu Y. A comparison of inner ear imaging features at different time points of sudden sensorineural hearing loss with three-dimensional fluid-attenuated inversion recovery magnetic resonance imaging. Eur Arch Otorhinolaryngol 2014 Aug 6. [Epub ahead of print]

65. Lee HY, Jung SY, Park MS, Yeo SG, Lee SY, Lee SK. Feasibility of three-dimensional fluid-attenuated inversion recovery magnetic resonance imaging as a prognostic factor in patients with sudden hearing loss. Eur Arch Otorhinolaryngol 2012; 269: 1885-1891.

66. Yoshida T, Sugiura M, Naganawa S, Teranishi M, Nakata S, Nakashima T. Three-dimensional fluidattenuated inversion recovery magnetic resonance imaging findings and prognosis in sudden sensorineural hearing loss. Laryngoscope 2008; 118:14331437.

67. Chung MS, Lee JH, Kim DY, et al. The clinical significance of findings obtained on 3D-FLAIR MR imaging in patients with Ramsay-Hunt syndrome. Laryngoscope 2014 Oct 27. [Epub ahead of print]

68. Sugiura M, Naganawa S, Nakata S, Kojima S, Nakashima T. 3D-FLAIR MRI findings in a patient with Ramsay Hunt syndrome. Acta Otolaryngol 2007; 127:547-549.

69. Nakata S, Mizuno T, Naganawa S, et al. 3D-FLAIR MRI in facial nerve paralysis with and without audio-vestibular disorder. Acta Otolaryngol 2010; 130: 632-636.

70. Sone M, Yoshida T, Naganawa S, et al. Comparison of computed tomography and magnetic resonance imaging for evaluation of cholesteatoma with labyrinthine fistulae. Laryngoscope 2012; 122:11211125 .

71. Yamazaki M, Naganawa S, Kawai H, Nihashi T, Fukatsu H, Nakashima T. Increased signal intensity of the cochlea on pre- and post-contrast enhanced
3D-FLAIR in patients with vestibular schwannoma. Neuroradiology 2009; 51:855-863.

72. Kim DY, Lee JH, Goh MJ, et al. Clinical significance of an increased cochlear 3D fluid-attenuated inversion recovery signal intensity on an MR imaging examination in patients with acoustic neuroma. AJNR Am J Neuroradiol 2014; 35:1825-1829.

73. Naganawa S, Satake H, Iwano S, Fukatsu H, Sone M, Nakashima T. Imaging endolymphatic hydrops at 3 tesla using 3D-FLAIR with intratympanic GdDTPA administration. Magn Reson Med Sci 2008; 7:85-91.

74. Fiorino F, Pizzini FB, Barbieri F, Beltramello A. Magnetic resonance imaging fails to show evidence of reduced endolymphatic hydrops in gentamicin treatment of Ménière's disease. Otol Neurotol 2012; 33:629-633.

75. Gurkov R, Flatz W, Louza J, Strupp M, Krause E. In vivo visualization of endolyphatic hydrops in patients with Meniere's disease: correlation with audiovestibular function. Eur Arch Otorhinolaryngol 2011; 268:1743-1748.

76. Chen X, Zhang XD, Gu X, Fang ZM, Zhang R. Endolymphatic space imaging in idiopathic sudden sensorineural hearing loss with vertigo. Laryngoscope 2012; 122:2265-2268.

77. Naganawa S, Satake H, Kawamura M, Fukatsu H, Sone M, Nakashima T. Separate visualization of endolymphatic space, perilymphatic space and bone by a single pulse sequence; $3 \mathrm{D}$-inversion recovery imaging utilizing real reconstruction after intratympanic Gd-DTPA administration at 3 Tesla. Eur Radiol 2008; 18:920-924.

78. Nakashima T, Naganawa S, Pyykko I, et al. Grading of endolymphatic hydrops using magnetic resonance imaging. Acta Otolaryngol Suppl 2009:5-8.

79. Fukuoka H, Tsukada K, Miyagawa M, et al. Semiquantitative evaluation of endolymphatic hydrops by bilateral intratympanic gadolinium-based contrast agent (GBCA) administration with MRI for Meniere's disease. Acta Otolaryngol 2010; 130: $10-16$.

80. Naganawa S, Yamazaki M, Kawai H, Bokura K, Sone M, Nakashima T. Estimation of perilymph enhancement after intratympanic administration of Gd-DTPA by fast $\mathrm{T}_{1}$-mapping with a dual flip angle 3D spoiled gradient echo sequence. Magn Reson Med Sci 2013; 12:223-228.

81. Seo YJ, Kim J, Choi JY, Lee WS. Visualization of endolymphatic hydrops and correlation with audiovestibular functional testing in patients with definite Meniere's disease. Auris Nasus Larynx 2013; 40: 167-172.

82. Naganawa S, Sugiura M, Kawamura M, Fukatsu H, Sone M, Nakashima T. Imaging of endolymphatic and perilymphatic fluid at 3T after intratympanic administration of gadolinium-diethylene-triamine pentaacetic acid. AJNR Am J Neuroradiol 2008; 29 : $724-726$. 
83. Katayama N, Yamamoto M, Teranishi M, et al. Relationship between endolymphatic hydrops and vestibular-evoked myogenic potential. Acta Otolaryngol 2010; 130:917-923.

84. Kato M, Teranishi M, Katayama N, Sone M, Naganawa S, Nakashima T. Association between endolymphatic hydrops as revealed by magnetic resonance imaging and caloric response. Otol Neurotol 2011; 32:1480-1485.

85. Suzuki H, Teranishi M, Naganawa S, Nakata S, Sone M, Nakashima T. Contrast-enhanced MRI of the inner ear after intratympanic injection of meglumine gadopentetate or gadodiamide hydrate. Acta Otolaryngol 2011; 131:130-135.

86. Sone $M$, Naganawa $S$, Teranishi $M$, Nakata $S$, Katayama N, Nakashima T. Changes in endolymphatic hydrops in a patient with Meniere's disease observed using magnetic resonance imaging. Auris Nasus Larynx 2010; 37:220-222.

87. Yamamoto M, Teranishi M, Naganawa S, et al. Relationship between the degree of endolymphatic hydrops and electrocochleography. Audiol Neurootol $2010 ; 15: 254-260$.

88. Teranishi M, Naganawa S, Katayama N, et al. Image evaluation of endolymphatic space in fluctuating hearing loss without vertigo. Eur Arch Otorhinolaryngol 2009; 266:1871-1877.

89. Yoshioka M, Naganawa S, Sone M, Nakata S, Teranishi M, Nakashima T. Individual differences in the permeability of the round window: evaluating the movement of intratympanic gadolinium into the inner ear. Otol Neurotol 2009; 30:645-648.

90. Fiorino F, Pizzini FB, Barbieri F, Beltramello A. Variability in the perilymphatic diffusion of gadolinium does not predict the outcome of intratympanic gentamicin in patients with Ménière's disease. Laryngoscope 2012; 122:907-911.

91. Kasai S, Teranishi M, Katayama N, et al. Endolymphatic space imaging in patients with delayed endolymphatic hydrops. Acta Otolaryngol 2009; 129: 1169-1174.

92. Naganawa $\mathrm{S}$, Ishihara $\mathrm{S}$, Iwano $\mathrm{S}$, Sone $\mathrm{M}$, Nakashima T. Three-dimensional (3D) visualization of endolymphatic hydrops after intratympanic injection of Gd-DTPA: optimization of a 3D-real inversion-recovery turbo spin-echo (TSE) sequence and application of a 32-channel head coil at 3T. J Magn Reson Imaging 2010; 31:210-214.

93. Naganawa $S$, Satake $H$, Iwano $S$, Sone $M$, Nakashima T. Communication between cochlear perilymph and cerebrospinal fluid through the cochlear modiolus visualized after intratympanic administration of Gd-DTPA. Radiat Med 2008; 26: 597-602.

94. Kawai H, Naganawa S, Ishihara S, Sone M, Nakashima T. MR imaging of the cochlear modiolus after intratympanic administration of Gd-DTPA. Magn Reson Med Sci 2010; 9:23-29.

95. Yamazaki M, Naganawa S, Kawai H, Sone M,
Nakashima T. Gadolinium distribution in cochlear perilymph: differences between intratympanic and intravenous gadolinium injection. Neuroradiology 2012; 54:1161-1169.

96. Shi H, Li Y, Yin S, Zou J. The predominant vestibular uptake of gadolinium through the oval window pathway is compromised by endolymphatic hydrops in Ménière's disease. Otol Neurotol 2014; 35: 315-322.

97. Liu F, Huang W, Chen Q, Meng X, Wang Z, He Y. Noninvasive evaluation of the effect of endolymphatic sac decompression in Ménière's disease using magnetic resonance imaging. Acta Otolaryngol 2014; 134:666-671.

98. Tagaya M, Yamazaki M, Teranishi M, et al. Endolymphatic hydrops and blood-labyrinth barrier in Ménière's disease. Acta Otolaryngol 2011; 131: 474-479.

99. Tagaya M, Teranishi M, Naganawa S, et al. 3 Tesla magnetic resonance imaging obtained 4 hours after intravenous gadolinium injection in patients with sudden deafness. Acta Otolaryngol 2010; 130: 665-669.

100. Nakashima T, Naganawa S, Teranishi M, et al. Endolymphatic hydrops revealed by intravenous gadolinium injection in patients with Ménière's disease. Acta Otolaryngol 2010; 130:338-343.

101. Naganawa S, Yamazaki M, Kawai H, Bokura K, Sone M, Nakashima T. Imaging of Ménière's disease after intravenous administration of single-dose gadodiamide: utility of multiplication of MR cisternography and HYDROPS image. Magn Reson Med Sci 2013; 12:63-68.

102. Tanigawa T, Tamaki T, Yamamuro O, et al. Visualization of endolymphatic hydrops after administration of a standard dose of an intravenous gadolinium-based contrast agent. Acta Otolaryngol 2011; 131:596-601.

103. Yoshida T, Teranishi M, Kato M, et al. Endolymphatic hydrops in patients with tinnitus as the major symptom. Eur Arch Otorhinolaryngol 2013; 270: 3043-3048.

104. Naganawa S, Yamazaki M, Kawai H, Bokura K, Sone M, Nakashima T. Three-dimensional visualization of endolymphatic hydrops after intravenous administration of single-dose gadodiamide. Magn Reson Med Sci 2013; 12:147-151.

105. Naganawa S, Yamazaki M, Kawai H, Bokura K, Sone M, Nakashima T. Imaging of Ménière's disease by subtraction of MR cisternography from positive perilymph image. Magn Reson Med Sci 2012; 11:303-309.

106. Naganawa S, Yamazaki M, Kawai H, Bokura K, Sone M, Nakashima T. Imaging of Ménière's disease after intravenous administration of single-dose gadodiamide: utility of subtraction images with different inversion time. Magn Reson Med Sci 2012; 11:213-219.

107. Naganawa S, Yamazaki M, Kawai H, Bokura K, 
Sone M, Nakashima T. Imaging of endolymphatic and perilymphatic fluid after intravenous administration of single-dose gadodiamide. Magn Reson Med Sci 2012; 11:145-150.

108. Naganawa S, Suzuki K, Nakamichi R, et al. Semi- quantification of endolymphatic size on MR imaging after intravenous injection of single-dose gadodiamide: comparison between two types of processing strategies. Magn Reson Med Sci 2013; 12:261269. 